\title{
A STUDY ON ALONG-TRACK AND CROSS-TRACK NOISE OF ALTIMETRY DATA BY MAXIMUM LIKELIHOOD: MARS ORBITER LASER ALTIMETRY (MOLA) EXAMPLE
}

\author{
Wojciech Jarmołowski, Jacek Łukasiak \\ Department of Satellite Geodesy and Navigation \\ Faculty of Geodesy, Geospatial and Civil Engineering \\ University of Warmia and Mazury \\ ul. Oczapowskiego 2 \\ 10-719 Olsztyn, Poland \\ e-mail: wojciech.jarmolowski@uwm.edu.pl; wojciech.jarmolowski@gmail.com \\ Phone +48895234764 \\ Fax +48895234723
}

\begin{abstract}
The work investigates the spatial correlation of the data collected along orbital tracks of Mars Orbiter Laser Altimeter (MOLA) with a special focus on the noise variance problem in the covariance matrix. The problem of different correlation parameters in along-track and crosstrack directions of orbital or profile data is still under discussion in relation to Least Squares Collocation (LSC). Different spacing in along-track and transverse directions and anisotropy problem are frequently considered in the context of this kind of data. Therefore the problem is analyzed in this work, using MOLA data samples. The analysis in this paper is focused on a priori errors that correspond to the white noise present in the data and is performed by maximum likelihood (ML) estimation in two, perpendicular directions. Additionally, correlation lengths of assumed planar covariance model are determined by ML and by fitting it into the empirical covariance function (ECF). All estimates considered together confirm substantial influence of different data resolution in along-track and transverse directions on the covariance parameters.
\end{abstract}

\section{Keywords}

LSC, noise, along-track, cross-track, maximum likelihood

\section{INTRODUCTION}

Many spaceborne, airborne and also ground techniques of data acquisition use a profile data collection, which is a natural consequence of the movement. The examples are airborne gravity (Olesen et al., 2000; Forsberg at al., 2001), bathymetry data (Hughes Clarke, 2003; Popielarczyk and Templin, 2014), sea altimetry (Andersen and Knudsen, 1998; Fok et al., 2010) and topography from the laser altimetry (Smith et al., 2001; McGovern et al., 2002), which is investigated in this paper. Terrestrial profile surveys, as well as satellite observations, always have a typical property of along-track data collection having several times larger point density than distances between the profiles (Dibarboure et al., 2013; Som et 
al., 2008). This is caused by different factors like time-consumption or costs; however, it may be concluded that there is no special cost of along-track densification if the vehicle is in a move and the sensors are efficient in respective time units. Therefore the spatial resolution is larger along the profiles than average resolution in all remaining directions. Some specific questions related to the data interpolation arise then due to this heterogeneity. How can a detailed regular grid be calculated from the data tracks in relation to the details available along the track? Should we use isotropic covariance function (CF) or anisotropic one and could the anisotropy be related with the data spatial density? This article provides some answers related to these issues.

Mars Orbiter Lunar Altimetry (MOLA) is an example of the satellite data with cross-track resolution significantly worse than along-track spacing (Smith et al., 2001; Neumann et al., 2001; Aharonson et al., 2000). MOLA topographic data is used in this article to investigate the problem of variable point data density in covariance matrix typical for Least Squares Collocation (LSC) (Moritz, 1980), which is, under some conditions, often regarded as close to simple kriging (Dermanis, 1984). The MOLA experiment has provided global topography of Mars, which is essential information in Mars exploration process. Accurate topography model, together with other data like e.g. gravity, constitutes the foundation for various geophysical investigations, such as volcanism, tectonics or past water transport (Smith et al. 2001). Therefore MOLA data were often analyzed in details, including hypsometry, slopes, correlation parameters and frequency power spectrum (Aharonson et al., 2000). The correlation was also studied using a spherical harmonic approach to separate different topography and gravity spectra, which are further used in the lithosphere studies (McGovern et al., 2002). The primary problem considered when working with the data tracks are crossover errors that have to be minimized by the least-squares adjustment (Neumann et al., 2001). This factor is also examined in this article, but treated in a simplified way, as the work is principally focused on another problem related with orbital data collection, i.e. different resolution in different spatial directions. In the case of MOLA, these resolutions are especially dependent on the latitude of observations. This dependence, as well as the resolution problem of created DEM models are discussed in Som et al. (2008).

Anisotropy is a property that is frequently investigated in LSC or kriging. This attribute is associated with non-stationarity of the spatial process and non-stationary kernels are developed to handle this problem in data modeling (Darbeheshti and Featherstone, 2009; Paciorek and Schervish, 2006). These kernels help in the modeling of the physical field in places where, due to different factors, different covariance parameters are required for different spatial directions. Sometimes, the differences between along-track errors and crosstrack errors coming from different resolutions are also considered as anisotropy (Dibarboure et al., 2013). Some authors investigate the errors in cross-track differences resulting from specific observational conditions by a method which applies different CF parameters for cross-track direction (Andersen and Knudsen, 1998). The above mentioned examples consider the influence of specific observational errors on covariance parameters heterogeneity, dependent on the azimuth. However, these papers also clearly demonstrate that the covariance is affected by the ratio between along-track and cross-track errors or sea surface variability, rather than by actual non-stationarity of the spatial field. Similar problems often lead to the use of other numerical techniques, applied at different steps of data processing like filtering (Kim et al., 2008; Matsumoto et al., 2000) or separate filtering in along-track direction only (Sandwell and Smith, 1997). This article follows the issues stated e.g. in Dibarboure et al. (2013) or Clark (2010), where a priori noise variance is implicitly associated with the sampling density, which is equivalent to the data spacing in case of altimetry data. Besides the along-track profiles that were ready to process, the cross-track profiles have been formed 
from the same MOLA data and both have been processed by maximum likelihood (ML) estimation. A priori errors representing data noise have been predicted profile-by-profile in both directions, together with correlation length (CL) of the adopted Gauss-Markov third order covariance model (GM3).

\section{ESTIMATION OF COVARIANCE PARAMETERS}

LSC with parameters can be represented by the known equation (Moritz, 1980)

$$
\mathbf{l}=\mathbf{X} \boldsymbol{\beta}+\mathbf{s}+\mathbf{n}
$$

where $\mathbf{l}$ is the vector of the observations, $\mathbf{s}$ is the stochastic part of the signal and $\mathbf{n}$ denotes the noise. $\mathbf{X} \boldsymbol{\beta}$ represents the deterministic part of the signal, which is of importance in LSC, because we need the condition of zero mean, also in local solutions, where the data mean is significantly moved from zero. Assuming now that our observations are heights, the necessary step is to remove deterministic trend, as follows:

$$
\mathbf{h}=\mathbf{X} \boldsymbol{\beta}+\mathbf{h}^{\mathrm{r}}
$$

where $\beta$ includes the vector of unknown trend parameters and $\mathbf{X}$ is design matrix of the trend, which can be based on

$$
\mathbf{X}=\left[\begin{array}{cccccc}
1 & \varphi_{1} & \lambda_{1} & \varphi_{1}{ }^{2} & \lambda_{1}{ }^{2} & \varphi_{1} \lambda_{1} \\
\cdots & \cdots & \cdots & \cdots & \cdots & \cdots \\
1 & \varphi_{n} & \lambda_{n} & \varphi_{n}{ }^{2} & \lambda_{n}{ }^{2} & \varphi_{n} \lambda_{n}
\end{array}\right]
$$

The trend is used in the so-called projection matrix realizing its removal in practice. The projection matrix $\mathbf{P}$ reads ( $n$ is the number of data):

$$
\mathbf{P}=\mathbf{I}_{n}-\mathbf{X}\left(\mathbf{X}^{\mathrm{T}} \mathbf{X}\right)^{-1} \mathbf{X}^{\mathrm{T}}
$$

Then detrended, residual part of the signal will be

$$
\mathbf{h}^{\mathrm{r}}=\mathbf{P h}
$$

The empirical covariance function (ECF) is an approximation of data covariance. It can be determined as a function of distance $\psi$, however the azimuth $\alpha$ is sometimes included, which generalizes the function to $2 \mathrm{D}$ representation. In the current work the ECF is calculated for constant azimuth, which in practice results in the intervals of the azimuth. The ECF is then

$$
\begin{gathered}
\forall(i, j) \quad \cos \psi=\cos \theta_{i} \cos \theta_{j}+\sin \theta_{i} \sin \theta_{j} \cos \left(\lambda_{i}-\lambda_{j}\right) \\
\wedge \quad \sin \alpha=\left(\sin \theta_{j} \sin \left(\lambda_{i}-\lambda_{j}\right)\right) / \sin \psi=\text { const. } \\
\quad \operatorname{ECF}\left(\psi, \alpha \mid \mathbf{h}^{\mathrm{r}}\right)=\frac{\sum_{i, j}^{k} \Delta h_{i}^{r} h_{j}^{r}}{k}
\end{gathered}
$$

where $\theta_{\mathrm{i}}=\pi / 2-\varphi_{\mathrm{i}}$ and $\psi$ is the spherical distance. Calculated values are grouped using intervals of uniformly increasing $\psi$. This test also includes groping in $\alpha$, i.e. in practice some interval of azimuths close to the selected one participates in the computation of the ECF representing individual direction in space. Usually, 2D representation of CF is calculated, i.e. the ECF for the full circle of azimuths is calculated. This work involves only two of them: an approximate azimuth of along-track direction and its cross section representing the shortest distances between original data tracks. The ECF provides local shapes of the covariance that have to be further modeled to be conveniently applied in LSC. There are many models used in 
LSC and kriging in practice, eg. GM3 model. This is an example of planar covariance model, however spherical distance $\psi$ is often used as an argument of the function, instead of planar one (Eq. 7):

$$
\begin{array}{r}
\forall(i, j) \quad \mid \quad \cos \psi=\cos \theta_{i} \cos \theta_{j}+\sin \theta_{i} \sin \theta_{j} \cos \left(\lambda_{i}-\lambda_{j}\right) \\
\wedge \sin \alpha=\left(\sin \theta_{j} \sin \left(\lambda_{i}-\lambda_{j}\right)\right) / \sin \psi=\text { const. } \\
G M 3\left(C_{0}, C L, \psi, \alpha\right)=C_{0}\left(1+\frac{\psi}{C L}+\frac{\psi^{2}}{3 \cdot C L^{2}}\right) \cdot \exp \left(\frac{-\psi}{C L}\right)
\end{array}
$$

$\mathrm{C}_{0}$ is often called "scale parameter" and is used here to represent the signal variance and CL is the correlation length. Three parameters described above compose the vector of covariance parameters $\boldsymbol{\theta}=\left[C_{0} ; C L ; \delta n\right]$. GM3 model can be used to determine the signal part of the covariance matrix $\mathbf{C}(\boldsymbol{\theta})$, which is composed of the signal and previously mentioned white noise, as follows:

$$
\mathbf{C}(\boldsymbol{\theta})=\mathbf{C}_{s}\left(C_{0}, C L\right)+\mathbf{C}_{n}(\delta n)
$$

The signal part $\mathbf{C}_{\mathrm{s}}$ is approximated solely by the covariance model (Eq. 7). The noise part $\mathbf{C}_{\mathrm{n}}$ can be modeled by the covariance model as well, in the occurrence of known or suspected correlation of the noise. In the case of white noise, it is defined by the variance only and reads

$$
\mathbf{C}_{n}(\delta n)=\delta n^{2} \mathbf{I}_{n}
$$

The parameters of the covariance matrix $\mathbf{C}(\boldsymbol{\theta})$ determine the spatial correlation of the signal and noise, which is necessary in LSC. Assuming now that the residuals are normally distributed, which is usually achievable locally after a successful removal of the longwavelength field, we may write probability density function (PDF) for a multivariate normal distribution based on the residuals $\mathbf{h}$, parameters $\boldsymbol{\beta}$ and covariance $\mathbf{C}(\boldsymbol{\theta})$ (Koch, 2007; Kusche, 2003; van Loon, 2008):

$$
p(\mathbf{h}, \boldsymbol{\theta})=(2 \pi)^{-\frac{n}{2}}|\mathbf{C}(\boldsymbol{\theta})|^{-\frac{1}{2}} \exp \left[-\frac{1}{2}(\mathbf{h}-\mathbf{X} \boldsymbol{\beta})^{T} \mathbf{C}(\boldsymbol{\theta})^{-1}(\mathbf{h}-\mathbf{X} \boldsymbol{\beta})\right]
$$

ML estimation starts with this assumption and aims at the maximization of PDF by selecting the covariance parameters. This maximization is simplified when using the logarithm of PDF from Eq. (10). Sometimes the preferred form of the method is restricted maximum likelihood (REML), but it is usually required for biased data (Searle et al., 1992; Jarmołowski and Bakuła, 2014). Since the data in the current test is residual after detrending, we decided to apply ML with no projection matrix in the ML equation. Sometimes it is more convenient to minimize the function with the opposite sign (Koch, 2007; van Loon, 2008; Searle et al., 1992). In this case the logarithm takes the form of negative log-likelihood function (NLLF) as follows:

$$
N L L F(\mathbf{h}, \boldsymbol{\theta})=-\ln p(\mathbf{h}, \boldsymbol{\theta})=\frac{n}{2} \ln (2 \pi)+\frac{1}{2} \ln |\mathbf{C}(\boldsymbol{\theta})|+\frac{1}{2}\left[(\mathbf{h}-\mathbf{X} \boldsymbol{\beta})^{T} \mathbf{C}(\boldsymbol{\theta})^{-1}(\mathbf{h}-\mathbf{X} \boldsymbol{\beta})\right]
$$

This minimization is resolved in the numerical part of the article by the selection of covariance parameters $\mathrm{CL}$ and $\delta$ after fixing $\mathrm{C}_{0}$, which is close to the real parameter in the vector of parameters $\boldsymbol{\theta}$, if the noise variance is of smaller order than the signal variance. 


\section{PRELIMINARY DATA SELECTION AND ASSESSMENT}

Two samples of MOLA footprints have been selected to perform the numerical experiment. The first set, from the northern hemisphere, is located on the slope of the crater Korolev and second, from the southern hemisphere, covers the region named Hellas Montes (Figs 1 and 2). As it was mentioned before, MOLA data footprints have different, irregular coverage due to the irregular separation between the tracks. Additionally, this separation is strongly related with the latitude and equatorial tracks are especially sparse in cross-track direction (Som et al., 2008). Moreover, significant crossover errors occur between the tracks globally. Global statistics show RMS at the level of $8 \mathrm{~m}$ before adjustment process (Neumann et al., 2001). The global adjustment has shown significant decrease of RMS to around $2 \mathrm{~m}$; however, in this work we decided to do no adjustment, since the crossovers can be significantly decreased by the removal of some data, which are redundant for this test. The crossovers are computed for both datasets and some tracks have shown values that are really outlying and useless for the adjustment. Therefore some tracks have been removed with the simultaneous control of the remaining crossover magnitude. The removal resulted in two advantages. Reduced datasets show more regular separation between the tracks with no overlaps in the sense of very close footprints. The RMS of crossovers in Korolev Crater sample after removal of outlying profiles is $1.8 \mathrm{~m}$ and that in the Hellas Montes is $3.1 \mathrm{~m}$. Since these results were close to the adjustment results from Neumann et al. (2001) and the cross-track separation has become more regular, the results were accepted. The outlying tracks with outlying crossover points are shown in red in Fig. 1.

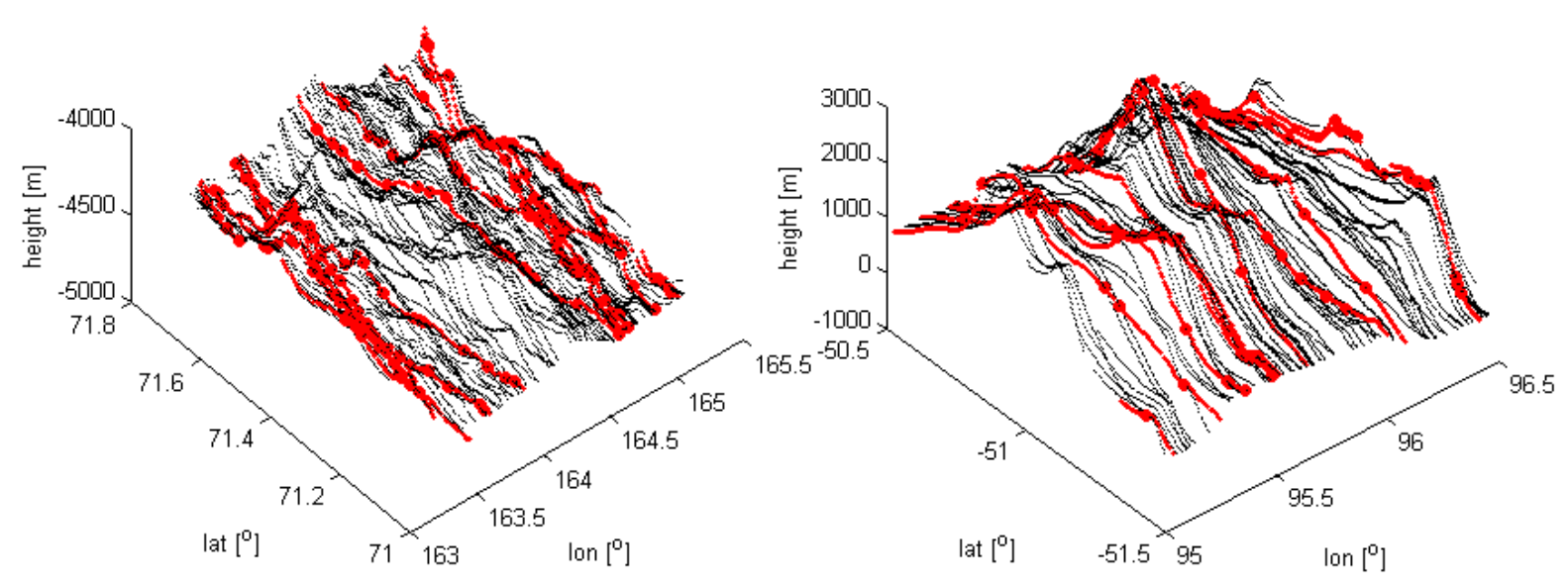

Fig. 1. Crossover analysis of data tracks. Reg large dots denote large crossovers (red tracks are removed).

Datasets cleaned of errors were then reduced for long-wavelength surface using second order polynomial trend (Eq. 3) and the projection matrix (Eq. 4). The removal of long wavelength surface is necessary for LSC process and for the estimation of the ECF, which is performed in the next section. Residual data are characterized by the zero mean and the variance, which is the total variance of the signal and noise at this step. The original data with the cleaned footprints of MOLA orbital tracks are shown in Fig. 2. 


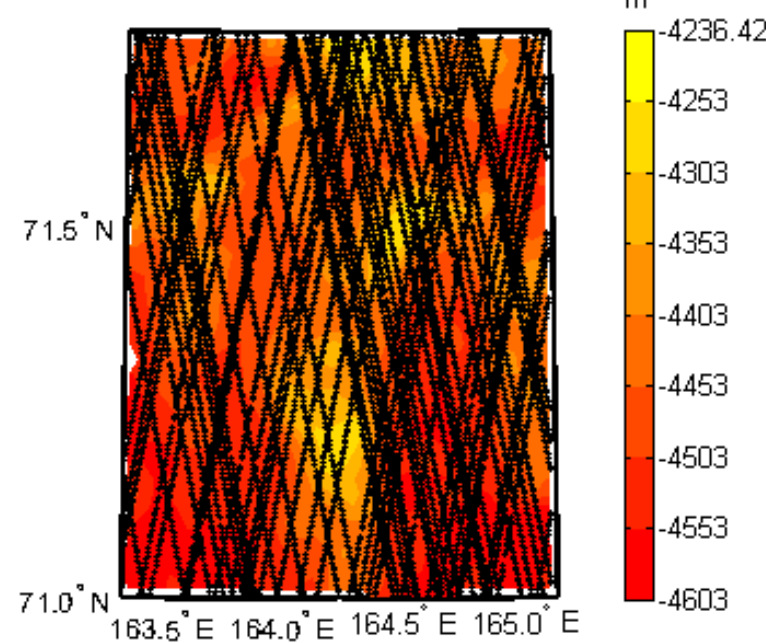

c) MOLA tracks over Hellas Montes

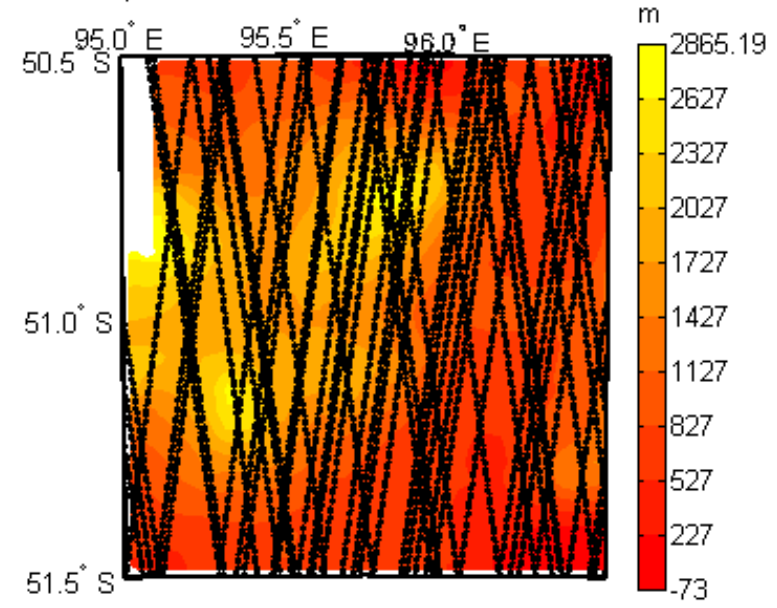

b) topography near Korolev Crater

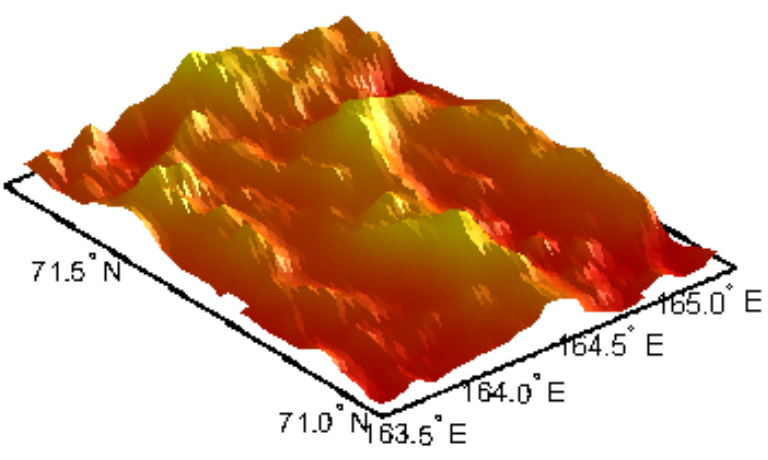

d) topography of Hellas Montes

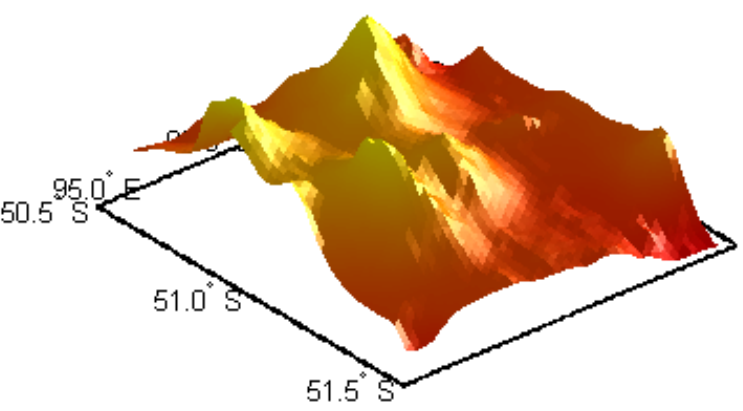

Fig. 2. Data tracks density and topography

\section{NUMERICAL TEST USING ALONG-TRACK AND CROSS-TRACK PROFILES}

Detrended and cleaned data are involved in a specific sampling process that is the key idea in the current work. The goal is to analyze the correlation only in two directions which are most often the focus of attention in relation to the orbital and profile data collection. These directions are along-track and cross-track (traverse) directions. Along-track profiles are easy to select, because the data is collected in separate orbital tracks provided with the numbers. Therefore the only action is to split them using these numbers, to obtain dense profiles of the points, ready for the calculation of individual ECFs in 1D, along the spherical distance. The average azimuths of these tracks have been computed to determine the azimuth of cross-track direction. These azimuths were $10.5^{\circ}$ for Korolev Crater and $7.5^{\circ}$ for the Hellas Montes. Figs $3 \mathrm{a}$ and $3 \mathrm{c}$ present all the tracks in red and the selected ones as black circles, drawn every third point to keep the figure esthetics. Cross-track direction is fixed as perpendicular to alongtrack profiles and the data collection has been performed by drawing great circles in this azimuth starting from all the points of the longest orbital track in the data sample. Then the closest data were selected for every particular great circle, using the threshold distance of $0.002^{\circ}$ from it. This has formed some number of cross-track profiles from the same dataset, but representing transverse azimuth and due to the separation between the original tracks, also different resolution. The points in the traverse profiles are aligned only approximately along 
the great circle, however this approximation is quite accurate due to the narrow threshold of $0.002^{\circ}$. The spacing between the points is also not homogeneous in transverse to along-track direction. Nevertheless, such irregularities cannot be eliminated from many datasets and therefore we decided to work including this property, as the natural data feature that has to be processed in practice. Figs $3 \mathrm{~b}$ and $3 \mathrm{c}$ present every fifth profile from the cross-track profiles to better visualize the rule of sampling.

a) Selected along-track profiles (Korolev Crater)

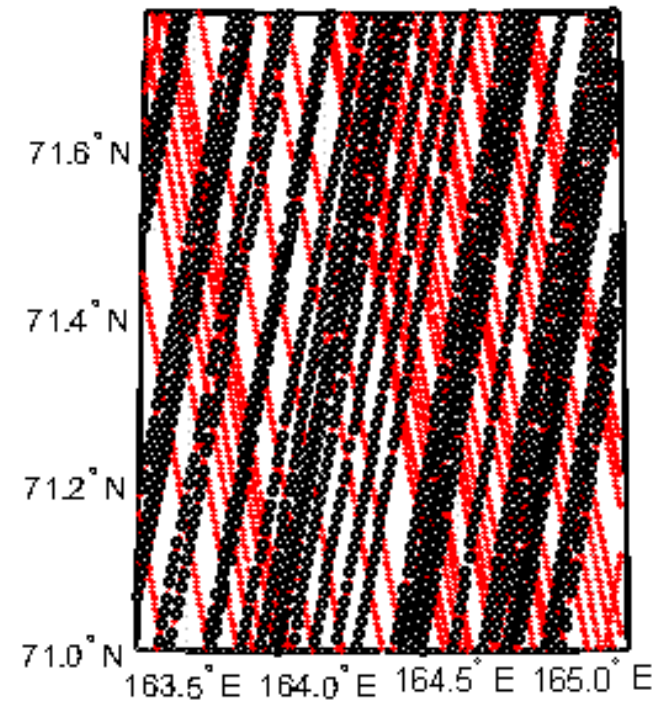

c) Selected along-track profiles (Hellas Montes)

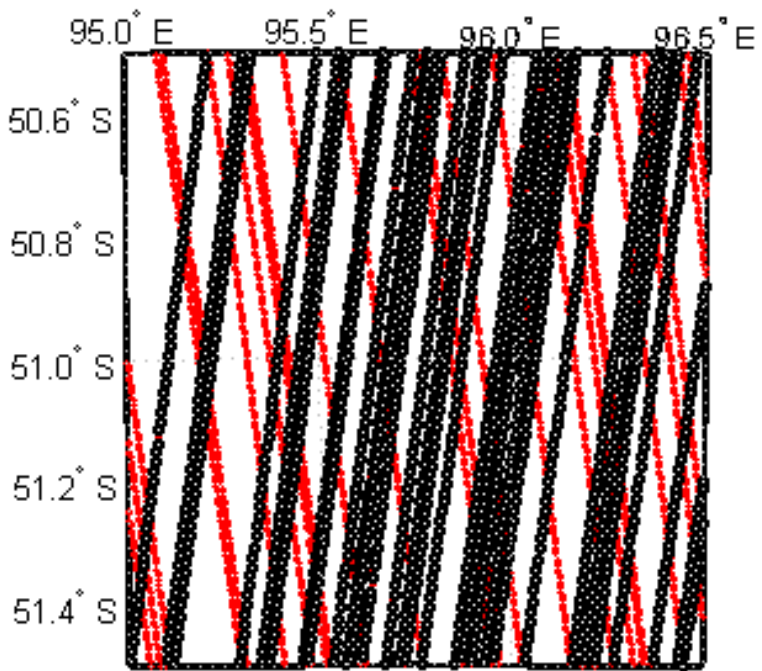

b) Data selected as cross-track (Korolev Crater)

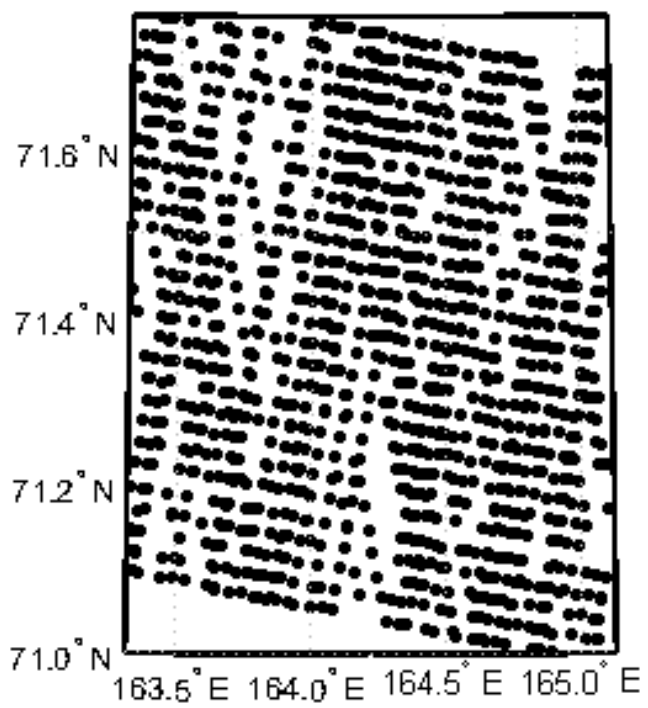

d) Data selected as cross-track (Hellas Montes)

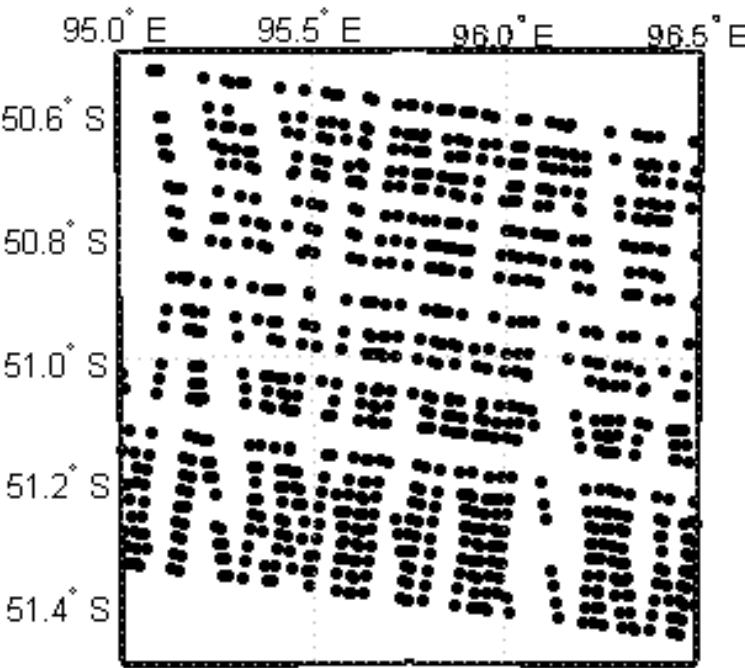

Fig. 3. Selection of along-track data - black circles drawn every third point in a) and c). Selection of cross-track data - black dots in b) and d) - all points in profiles, but every fifth profile drawn for clarity.

Three parameters are a subject of the investigation. Two parameters are usually easy to attain from the fitting of analytical planar covariance model to the ECF, which is here estimated in $1 \mathrm{D}$ along the profiles and averaged for the same direction. These parameters are data variance $\left(\mathrm{C}_{0}\right)$, which in case of relatively small noise variance is usually close to the signal variance and correlation length (CL) (Eq. 7). Two parameters were also inferred using ML method 
from the same data profiles. CL is estimated once again by ML and noise variance ( $\delta n)$ is assessed solely by ML, as its value is hard to assess from the CF fitting, because the shape of the ECF strongly depends on the data number and sampling density (Figs $4 \mathrm{~b}$ and $4 \mathrm{~d}$ ). The ECF is calculated approximately in 1D assuming constant azimuth, using separately points from individual profiles and spherical distances between the points. The approximation results from the approximate values of the azimuths between individual points in profiles. Average ECFs are then calculated by averaging the values at the same $\psi$ in different profiles (Fig. 4). The sampling interval of $\psi=0.01^{\circ}$ in the along-track direction (Eq. 6) is based on the average spacing of points along the original (along-track) profiles, which equals $0.005^{\circ}$. The use of the half of the average spacing distance prevents the lack of ECF pairs at shorter distances. A Cross-track sampling interval also starts from $0.01^{\circ}$ and is increased if the ECF shape is unstable, especially at shorter distances. This influence of the sampling distance on the ECF shape at short distances is particularly inconvenient in assessing noise from CF fitting. The GM3 model shows good coincidence with ECF values for used topography residuals in case of regular sampling in along-track direction. Cross-track estimation reveals more ECF oscillation, which can be implied by remaining crossover errors or variable data spacing. Nevertheless, the estimated $\mathrm{C}_{0}$ and $\mathrm{CL}$ values are similar for both datasets and no significant field anisotropy can be observed here.
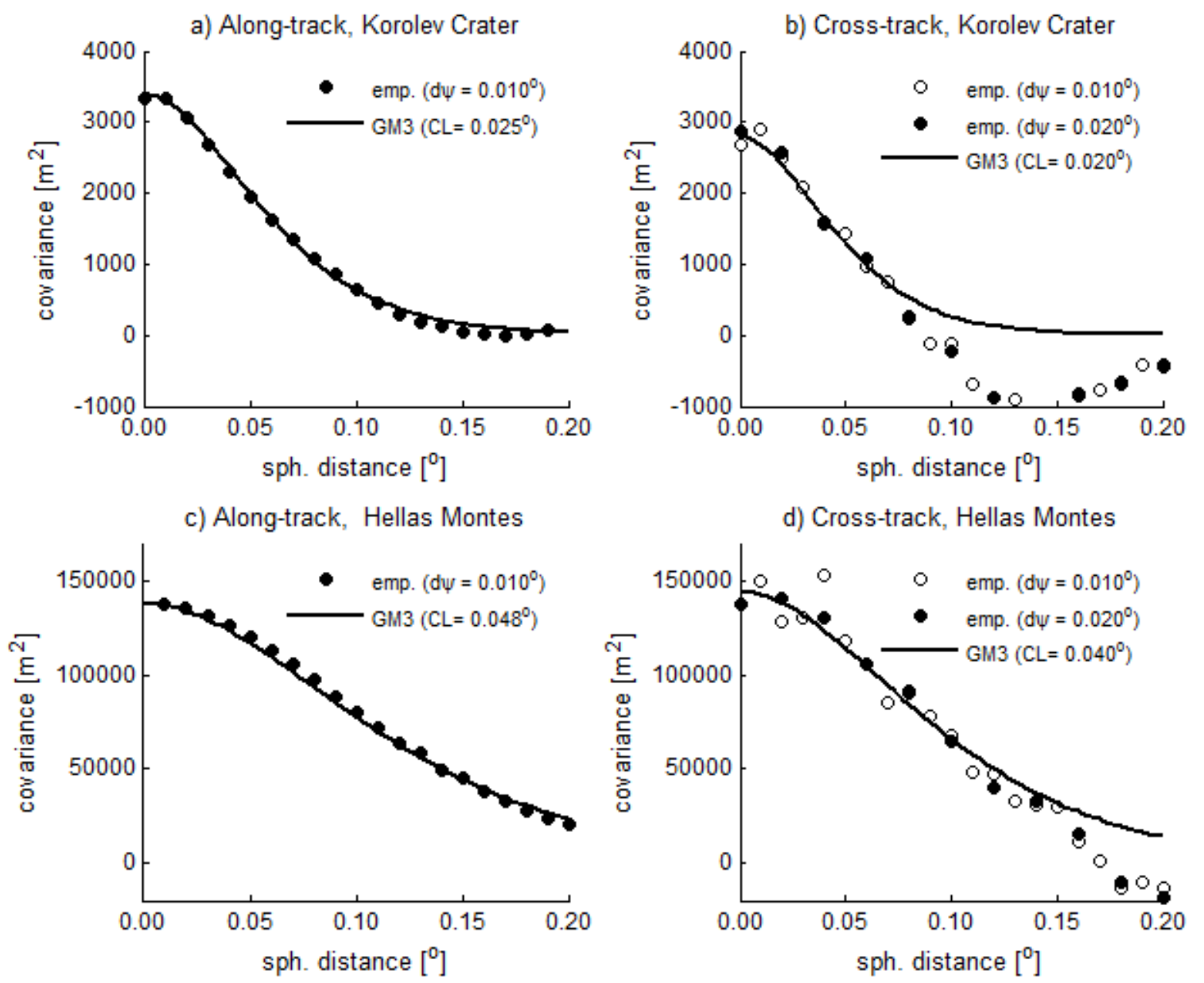

Fig. 4. Averages of ECFs for along-track profiles (a and c) and for cross-track profiles (b and d) and GM3 fitted. 
The non-homogeneity of the separation between the points in traverse profiles and other factors contribute to changes in ECF, which is especially visible in Fig. 4b. The influence of the mentioned property on ML estimation results was not known before the test. Therefore the main advantage is that the approach is in some sense comparative, due to the simultaneous use of along-track profiles with the equal spacing between the points. The covariance matrices based on the residual data (Eq. 8) are computed separately for individual data profiles in both directions. These matrices are applied in Eq. (11) to calculate NLLF minima using some range of CL and $\delta$ n (Table 1).

Table 1. The scheme of parameters ranges in ML estimation.

\begin{tabular}{|c|c|c|c|c|c|}
\hline & & \multicolumn{2}{|r|}{ Along-track } & \multicolumn{2}{|r|}{ Cross-track } \\
\hline & & $\delta \mathrm{n}$ in $\mathrm{m}$ & CL in degrees & $\delta \mathrm{n}$ in $\mathrm{m}$ & CL in degrees \\
\hline \multirow{3}{*}{ Korolev Crater } & Min. & 0.1 & 0.0050 & 0.1 & 0.0050 \\
\hline & Step & 0.5 & 0.0025 & 1.0 & 0.0050 \\
\hline & Max. & 4.1 & 0.0400 & 10.1 & 0.0800 \\
\hline \multirow{3}{*}{ Hellas Montes } & Min. & 0.1 & 0.0050 & 0.1 & 0.0050 \\
\hline & Step & 0.5 & 0.0050 & 1.0 & 0.0050 \\
\hline & Max. & 4.1 & 0.0800 & 12.1 & 0.1200 \\
\hline
\end{tabular}

The ranges in Table 1 were selected empirically, having an approximation of CL from the fitting of GM3 model into ECF shape and the knowledge of a typical MOLA data error (Smith et al., 2001; Aharonson et al., 2000). The signal variance $C_{0}$ has been fixed to be equal to its approximation by the ECF (Fig. 4). We have assumed that its inaccuracy resulting from the nature of the fitting can have only a few orders smaller impact on $\delta$ n and therefore NLLF values can properly indicate the parameters that are most efficient for LSC solution (Jarmołowski and Bakuła, 2014). The example plots of NLLF values for one along-track and one cross-track from the Hellas Montes data are shown in Fig. 5.
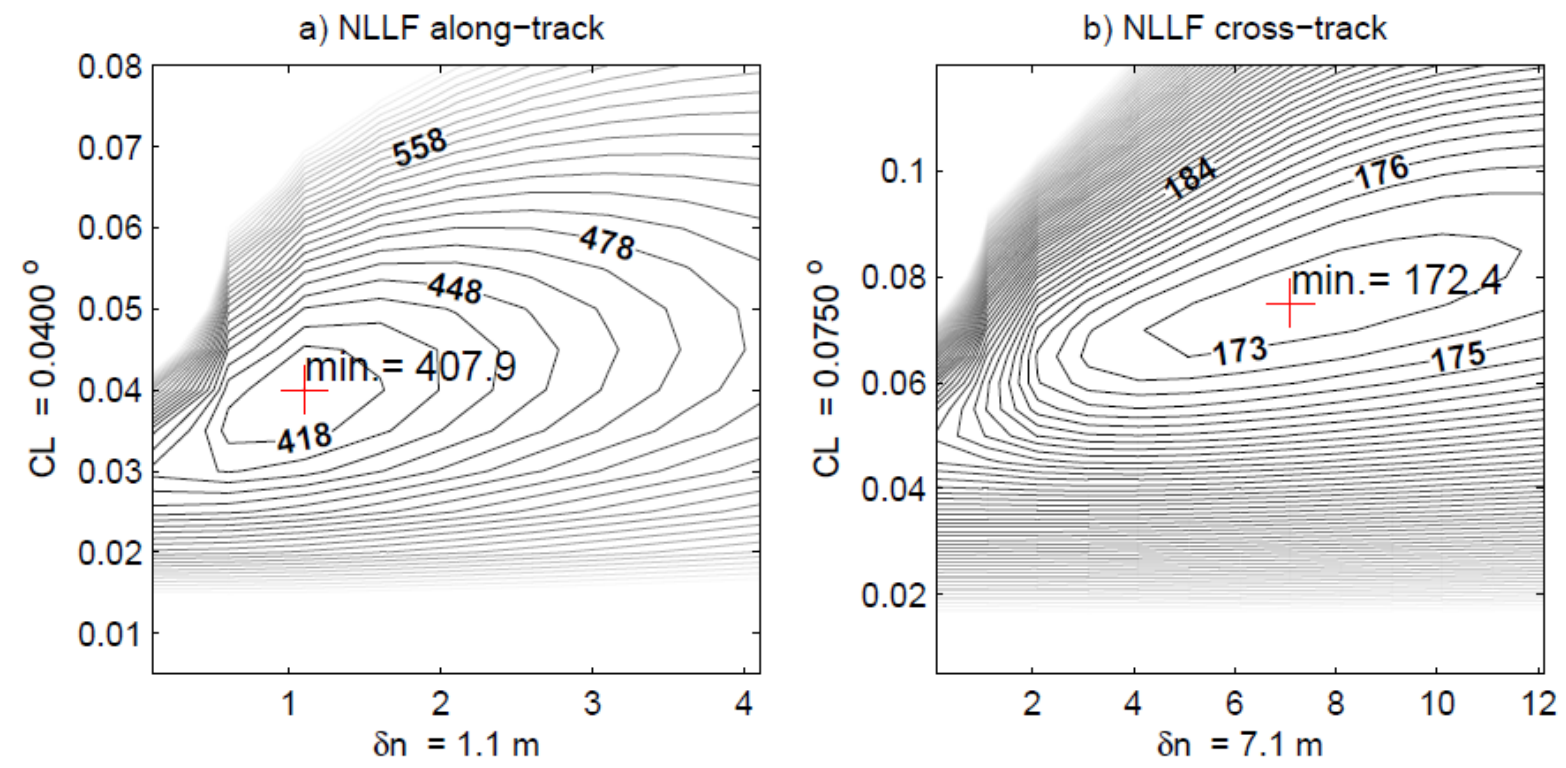

Fig. 5. Examples of NLLF calculation of covariance parameters for one along-track profile (a) and one cross-track profile (b).

The values of CL and $\delta n$ that realize the minimum of NLLF for individual covariance matrices are presented together in Fig. 6, in the same order as the profiles. The number of cross-track profiles is always larger than along-track and this is visible in Fig. 6. Bold lines with circles represent the parameters for individual original profiles (along-track) estimated 
by the minima of NLLF. The order of the profiles is geographically random and depends on the orbit numbers. The a priori errors $\delta$ are very similar for both datasets, i.e. between 1 and $3 \mathrm{~m}$, which is presented in Figs $6 \mathrm{a}$ and $6 \mathrm{c}$ respectively. This corresponds quite well to the MOLA accuracy assumed in the literature (Smith et al., 2001; Aharonson et al., 2000), however the resolution in along-track direction is also an important factor (Dibarboure et al., 2013; Clark, 2010; Jarmołowski, 2013). Comparing known along-track density at the level of 300-400 m with the power spectrum graph from Aharonson et al. (2000), it may be found that the topography from MOLA represents a few meters variability of the signal at the frequency of a few hundred meters. CL values for the along-track directions are two times larger for the Hellas Montes (Fig 6d). However, the computation of CL by ML for both datasets is compatible with CL from the ECF (4a and 4c).
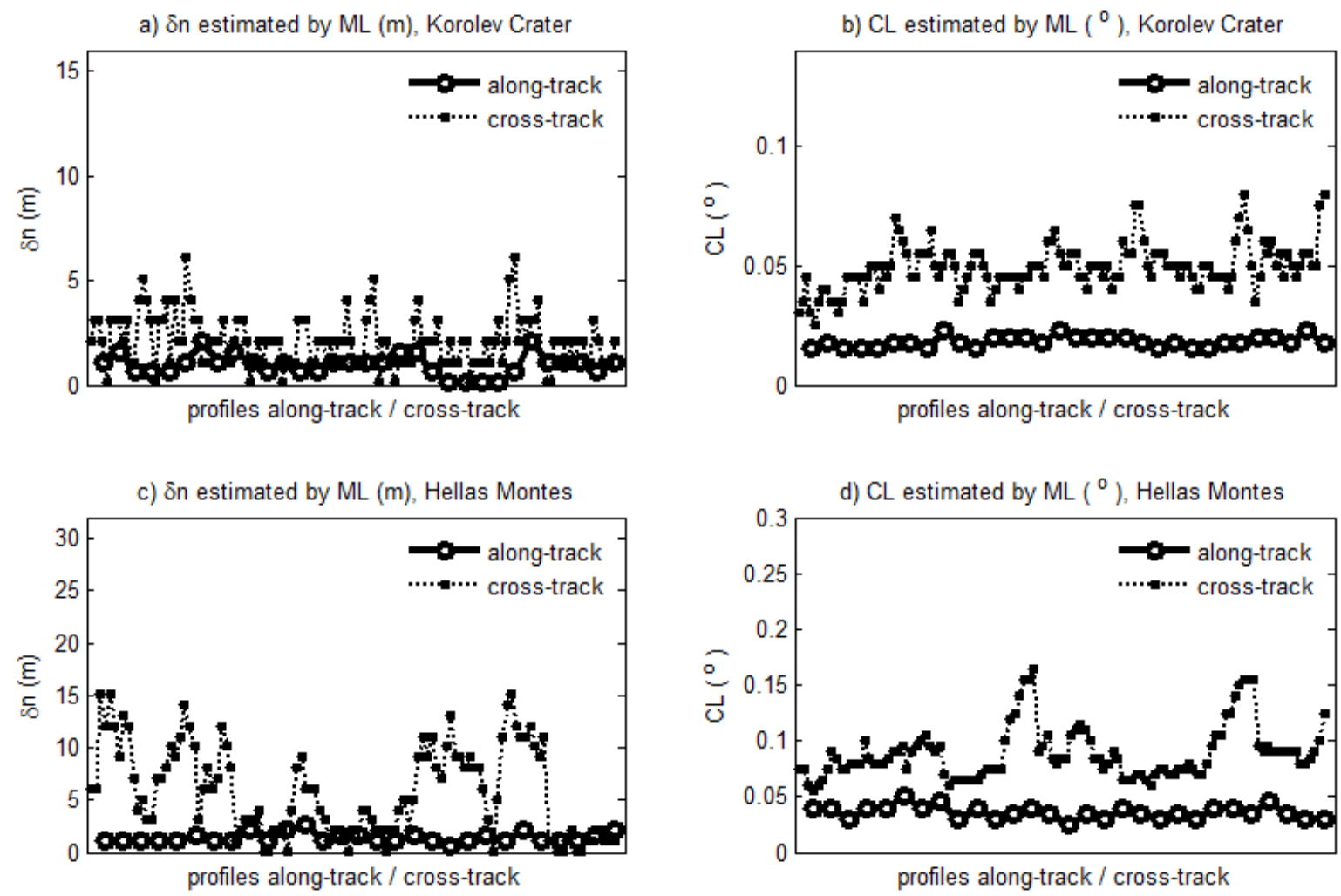

Fig. 6. ML estimation of covariance parameters for individual along-track profiles (solid line with circles) and cross-track profiles (dotted line with dots).

The change of the parameters in traverse direction was expected, but as it was pointed above, the effect of irregularity in the data resolution on ML estimates is not known. Therefore the combined influence from lower data resolution, irregularity and probably remaining crossover errors is observed in the parameters estimation results by ML inference. Consequently, in this article, rather natural than idealized conditions are tested in cross-track profiles and their comparison with the original profiles can give a preliminary assessment of the supposed anisotropy problems. The cross-track data distribution results in 2-3 times larger $\delta$ for Korolev data (Fig 6a) and several times larger for the Hellas Montes (Fig 6c). These differences were expected, because cross-track density is significantly lower for the Hellas Montes than for Korolev Crater due to the latitude, which naturally results in a different a priori error (Dibarboure et al., 2013). Singular small values of $\delta$ that are close to zero are specific ML estimation problems resulting from the spacing, trend model, covariance model applied and possibly other various factors that are still under consideration by the statisticians (Mardia, 2007). The comparative approach, which is the simultaneous estimation for regular and irregular data spacing is helpful, because the same algorithm is tested with regularly and 
irregularly spaced data. The irregular, sparse spacing in traverse direction also affects CL parameter estimated by ML. The NLLF requires two times larger CL for both data regions to attain the minima, than CL from the function fitting (Figs $6 b$ and $6 c$ ). This effect is confirmed for all traverse profiles.

\section{DISCUSSION AND CONCLUSIONS}

The comparative approach allows for some control of applied algorithms primarily on the regular, dense data along the orbital footprints. CL estimated in along-track directions is quite consistent with the results from $\mathrm{CF}$ fitting and $\delta$ corresponds to known errors of MOLA data. The minima of NLLF suggest increasing $\delta n$ and CL for cross-track direction. We see three substantial differences between along-track and cross-track data profiles. Cross-track profiles have remaining crossover errors, lower data resolution and irregular distribution. Since the crossovers are close to obtained $\delta$ for along-track direction, the remaining two factors are mostly suspected to be enforcing larger parameters for GM3 model. The lower data distribution results in the loss of higher spectral frequencies, i.e. terrain details of the resolution higher than data resolution. This contributes to a priori error budget. Irregularity of the cross-track data results in varying $\delta$ parameter (Figs 6b and 6d) in comparison to the relatively constant estimate for along-track profiles (Figs 6a and 6c). The CL estimates by ML for cross-track data are larger than those from CF fitting and ML estimates for along-track direction. The conclusions are not so straightforward here, however, it is possible that a larger CL incorporates additional data, which gives some improvement. To summarize, the change of resolution results in $\delta \mathrm{n}$ and CL increase and therefore the profile data and orbital tracks has to include this property in the processing. Manipulating the a priori noise variance in different directions may be worth considering in data processing, if it can preserve some information in comparison to data filtering.

\section{ACKNOWLEDGEMENTS}

MOLA data were downloaded from PDS Geosciences Node at Washington University in St. Louis.

\section{REFERENCES}

Aharonson, O., Zuber, M.T., Rothman, D.H., 2000. Statistics of Mars' topography from MOLA: Slopes, correlations and physical models. Journal of Geophysical Research 106(E10), 23723-23736.

Andersen, O.B., Knudsen, P., 1998. Global marine gravity field from the ERS-1 and Geosat geodetic mission altimetry. Journal of Geophysical Research 103 (C4): 8129-8137.

Clark, I., 2010. Statistics or geostatistics? Sampling error or nugget effect? Journal of the Southern African Institute of Mining and Metallurgy 110, 307-312.

Darbeheshti, N., Featherstone, W.E., 2009. Non-stationary covariance function modelling in 2D least squares collocation. Journal of Geodesy 83(6): 495-508.

Dermanis, A., 1984. Kriging and Collocation - A Comparison. Manuscripta Geodaetica 9 (3), 159-167.

Dibarboure, G., Le Traon P.Y., Galin N., 2013. Exploring the Benefits of Using CryoSat-2's Cross-Track Interferometry to Improve the Resolution of Multisatellite Mesoscale Fields. Journal of Atmospheric and Oceanic Technology 30, 1511-1526. 
Fok, H.S., Baki Iz H., Shum, C.K., Yi Y., Andersen, O., Braun, A., Yi C., Han, G., Kuo, C.Y., Matsumoto, K., Tony Song, Y., 2010. Evaluation of Ocean Tide Models Used for Jason-2 Altimetry Corrections. Marine Geodesy 33(1), 285-303.

Forsberg, R., Olesen, A.V., Keller, K., Møller, M., Gidskehaug A., Solheim, D., 2001. Airborne gravity and geoid surveys in the Arctic and Baltic seas. Proceedings of International Symposium on Kinematic Systems in Geodesy, Geomatics and Navigation (KIS-2001), Banff, pp. 586-593.

Hughes Clarke, J.E., 2003. Dynamic motion residuals in swath sonar data: Ironing out the creases. International Hydrographic Review 4(1):6-23.

Jarmołowski, W., 2013. A priori noise and regularization in least squares collocation of gravity anomalies. Geodesy and Cartography 62(2), 199-216.

Jarmołowski, W., Bakuła, M., 2014. Precise estimation of covariance parameters in leastsquares collocation by restricted maximum likelihood. Studia Geophysica et Geodaetica 58, 171-189.

Kim, J.W., Roman D.R., Lee B.Y., Kim Y., 2008. Altimetry Enhanced Free-air Gravity Anomalies in the High Latitude Region. Terrestrial, Atmospheric and Oceanic Sciences 19(1-2), 111-116.

Koch, K.R., 2007. Introduction to Bayesian Statistics. Second Edition. Springer-Verlag, Berlin, Germany.

Kusche, J., 2003. A Monte-Carlo technique for weight estimation in satellite geodesy. Journal of Geodesy 76, 641-652.

Mardia, K.V., 2007. Should geostatistics be model-based? In: Chen Q., Zhao P. and Agterberg F. (Eds.), Proceedings of the IAMG 2007 Conference: Geomathematics and GIS Analysis of Resources, Environment and Hazards. International Association for Mathematical Geosciences, Houston, TX (http://www1.maths.leeds.ac.uk/ sta6kvm/IAMGBeijingFinal.pdf).

McGovern, P.J., Solomon S.C., Smith D.E., Zuber M.T., Simons M., Wieczorek M.A., Phillips R.J., Neumann G.A., Aharonson O., Head J.W., 2002. Localized gravity/topography admittance and correlation spectra on Mars: Implications for regional and global evolution, Journal of Geophysical Research 107(E12), 5136, doi:10.1029/2002JE001854.

Matsumoto, K., Takanezawa, T., Ooe M., 2000. Ocean Tide Models Developed by Assimilating TOPEX/ POSEIDON Altimeter Data into Hydrodynamical Model: A Global Model and a Regional Model around Japan, Journal of Oceanography 56, 567-581.

Moritz, H., 1980. Advanced Physical Geodesy. Herbert Wichmann Verlag, Karlsruhe.

Neumann, G.A., Rowlands D.D., Lemoine F.G., Smith D.E., Zuber M.T., 2001. Crossover analysis of Mars Orbiter Laser Altimeter data. Journal of Geophysical Research 106 (E10), 23753-23768.

Olesen, A.V., Forsberg, R., Keller, K., Gidskehaug, A., 2000. Airborne gravity survey of Lincoln sea and Wandel Sea, North Greenland. Physics and Chemistry of the Earth, Part A: Solid Earth and Geodesy, 25(1), 25-29.

Paciorek, C.J., Schervish, M.J., 2006. Spatial modelling using a newclass of non-stationary covariance functions. Environmetrics 17(5), 483- 506. 
Popielarczyk, D., Templin, T., 2014. Application of Integrated GNSS/Hydroacoustic Measurements and GIS Geodatabase Models for Bottom Analysis of Lake Hancza: the Deepest Inland Reservoir in Poland, Pure and Applied Geophysics 171, 997-1011.

Sandwell, D.T., Smith, W.H.F., 1997. Marine gravity anomaly from Geosat and ERS-1 satellite altimetry. Journal of Geophysical Research 102, 10039-10054.

Searle, S.R., Casella, G., McCulloch, C.E., 1992. Variance Components, Wiley, New York.

Smith, D.E., et al. 2001. Mars Orbiter Laser Altimeter: Experiment summary after the first year of global mapping of Mars. Journal of Geophysical Research 106(E10), 2368923722, doi:10.1029/2000JE001364.

Som, S.M., Greenberg, H.M., Montgomery, D.R., 2008. The Mars Orbiter Laser Altimeter dataset: Limitations and improvements. Mars 4, 14-26, doi:10.1555/mars.2008.0002.

van Loon, J., 2008. Functional and stochastic modelling of satellite gravity data. $\mathrm{PhD}$ thesis, Delft University of Technology.

Received: 2015-05-05,

Reviewed: 2015-10-26, by E. K. Paršeliūnas,

Accepted: 2015-11-19. 